\title{
High prevalence of multidrug resistant ESBL- and plasmid mediated AmpC- producing clinical isolates of Escherichia coli at Maputo Central Hospital, Mozambique
}

Calvina E. L. Estaleva1,2,3, Tomas F. Zimba ${ }^{1,2}$, John Osei Sekyere³, Usha Govinden³, Hafizah Y. Chenia ${ }^{4}$, Gunnar S. Simonsen ${ }^{3,5,6}$, Bjørg Haldorsen ${ }^{5}$, Sabiha Y. Essack ${ }^{3}$ and Arnfinn Sundsfjord ${ }^{3,5,6^{*}}$ (i)

\begin{abstract}
Background: Epidemiological data of cephalosporin-resistant Enterobacterales in Sub-Saharan Africa is still restricted, and in particular in Mozambique. The aim of this study was to detect and characterize extended-spectrum $\beta$ lactamase (ESBL) - and plasmid-mediated AmpC (pAmpC)-producing clinical strains of Escherichia coli at Maputo Central Hospital (MCH), a 1000-bed reference hospital in Maputo, Mozambique.

Methods: A total of 230 clinical isolates of E. coli from urine $(n=199)$ and blood cultures $(n=31)$ were collected at MCH during August-November 2015. Antimicrobial susceptibility testing was performed by the disc diffusion method and interpreted according to EUCAST guidelines. Isolates with reduced susceptibility to 3rd generation cephalosporins were examined further; phenotypically for an ESBL-/AmpC-phenotype by combined disc methods and genetically for ESBL- and PAmpC-encoding genes by PCR and partial amplicon sequencing as well as genetic relatedness by ERIC-PCR.

Results: A total of 75 isolates with reduced susceptibility to cefotaxime and/or ceftazidime $(n=75)$ from urine $(n=$ 58/199; 29\%) and blood $(n=17 / 31 ; 55 \%)$ were detected. All 75 isolates were phenotypically ESBL-positive and 25/75 (33\%) of those also expressed an AmpC-phenotype. ESBL-PCR and amplicon sequencing revealed a majority of bla $a_{\text {CTX-M }}(n=58 / 75 ; 77 \%)$ dominated by bla positive for one or more PAmpC-genes dominated by bla Mox/FOx. Multidrug resistance (resistance $\geq$ three antibiotic classes) was observed in all the 75 ESBL-positive isolates dominated by resistance to trimethoprimsulfamethoxazole, ciprofloxacin and gentamicin. ERIC-PCR revealed genetic diversity among strains with minor clusters indicating intra-hospital spread.

(Continued on next page)
\end{abstract}

\footnotetext{
*Correspondence: arnfinn.sundsfjord@uit.no

${ }^{3}$ Antimicrobial Research Unit, School of Health Sciences, University of KwaZulu-Natal, Durban, South Africa

${ }^{5}$ Department of Microbiology and Infection Control, Norwegian National Advisory Unit on Detection of Antimicrobial Resistance, University Hospital of North Norway, Tromsø, Norway

Full list of author information is available at the end of the article
} 
(Continued from previous page)

Conclusion: We have observed a high prevalence of MDR pAmpC-and/or ESBL-producing clinical E. coli isolates with FOX/MOX and CTX-Ms as the major $\beta$-lactamase types, respectively. ERIC-PCR analyses revealed genetic diversity and some clusters indicating within-hospital spread. The overall findings strongly support the urgent need for accurate and rapid diagnostic services to guide antibiotic treatment and improved infection control measures.

Keywords: Escherichia coli, Multidrug resistance, Extended-spectrum $\beta$-lactamase, Plasmid-mediated AmpC $\beta$ lactamase

\section{Background}

The World Health Organization recognizes antimicrobial resistance (AMR) as a major global public health problem $[1,2]$. The dissemination of multidrug-resistant (MDR), extended-spectrum $\beta$-lactamase (ESBL)-producing Enterobacterales is of particular concern limiting treatment options for invasive infections to last line antibiotics [3-5].

ESBLs are enzymes that hydrolyze an extended spectrum of $\beta$-lactam antibiotics including penicillins and oxyimino-cephalosporins, but not cephamycins [6]. The most widely distributed ESBL enzymes are CTX-Mtype $\beta$-lactamases, which preferentially hydrolyze cefotaxime. The worldwide dissemination of CTX-Mtype $\beta$-lactamases has been dramatic and greater than the impact of the TEM- and SHV-type ESBLs [5, 7].

Plasmid-mediated AmpC $\beta$-lactamases (pAmpCs) are also an important cause of broad spectrum $\beta$-lactam resistance in Gram-negative bacteria [8]. Many Gramnegative bacteria including most Enterobacterales have chromosomally-encoded AmpC- $\beta$-lactamases. Some AmpC-genes have been mobilized from their chromosomal origin and may be spread by plasmids (pAmpC), with $b l a_{\text {MOX/FOX/DHA/CMY }}$ being the most prevalent variants $[6,8,9]$. In general, AmpC $\beta$-lactamases confer resistance to a wide range of $\beta$-lactam drugs including penicillins, cephamycin, 1 st - 3rd generation cephalosporins as well as classical $\beta$-lactamase inhibitors like clavulanic acid and tazobactam to which ESBLs are sensitive [6]. Co-presence of ESBLs and pAmpCs may occur [10].

In the context of low- and medium income countries (LMICs), and sub-Saharan Africa in particular, there are knowledge gaps concerning the epidemiology of ESBLand pAmpC-producing Enterobacterales in different ecological settings [11-13]. This study delineates plasmid AmpC- and ESBL-mediated resistance in clinical isolates of E. coli processed at the Maputo Central Hospital, Mozambique, in 2015.

\section{Methods}

\section{Bacterial isolates}

The strain collection consisted of isolates recovered from clinical specimens referred to the Department of
Clinical Microbiology, Maputo Central Hospital, a 1000bed reference hospital in Maputo, Mozambique. We included urine and blood culture samples from midAugust through mid-November 2015. Clinical information was obtained from the laboratory request form only, allowing distinction between in- and outpatients. Urine samples were obtained from both in- and outpatient whereas blood cultures were from inpatients only. Only one isolate per patient was included in the study.

\section{Bacterial species identification and Antimicrobial Susceptibility Testing (AST)}

E. coli strains were identified by standard biochemical tests including the API-20E kit (bioMerieux, la Balmeles-Grottes, France). AST was performed using the disk diffusion method according to EUCAST [14] and corresponding 2015-guidelines for interpretation including ampicillin $(10 \mu \mathrm{g})$, amoxicillin-clavulanic acid $(20-10 \mu \mathrm{g})$, piperacillin-tazobactam $(30-6 \mu \mathrm{g})$, cefoxitin $(30 \mu \mathrm{g})$, cefotaxime $(5 \mu \mathrm{g})$, ceftazidime $(10 \mu \mathrm{g})$, ceftriaxone $(30 \mu \mathrm{g})$, meropenem $(10 \mu \mathrm{g})$, ciprofloxacin $(5 \mu \mathrm{g})$, gentamicin $(10 \mu \mathrm{g})$, trimethoprim-sulfamethoxazole $(1.25-23.75 \mu \mathrm{g})$, and nitrofurantoin $(100 \mu \mathrm{g})$. MDR was defined as acquired resistance to at least one agent in three or more antimicrobial classes [15].

Quality assurance was performed weekly during the study period using the wild-type E. coli ATCC 25922 strain. Moreover, K. pneumoniae ATCC 700603 (ESBLtype $b l a_{\mathrm{SHV}-18}$ ) and E.coli A5-8 (pAmpC-type bla $a_{\mathrm{DHA}}$ ) were used as ESBL- and AmpC-positive controls, respectively. E. coli A5-8 is a well characterized strain that was obtained from the Norwegian Advisory Unit for Detection of Antimicrobial Resistance. Strains were stored in skimmed milk broth with $10 \%$ glycerol at $-70{ }^{\circ} \mathrm{C}$ until molecular analysis.

\section{Phenotypic detection of $\beta$ - lactamases}

Isolates that showed reduced susceptibility (I or R) to cefotaxime and/or ceftazidime were examined for ESBLproduction. Moreover, isolates with reduced susceptibility to cefotaxime and/or ceftazidime and cefoxitin were examined for increased AmpC-production [16]. The mechanisms of resistance were examined using ROSCO combined disc tablets (Rosco Diagnostic, Denmark) for 
ESBL and/or increased AmpC-production and interpreted according to manufacturer's instructions [17].

\section{Genetic detection and characterization of $\beta$-lactamases}

Genetic characterization was performed at the Antimicrobial Research Unit, University of KwaZulu-Natal (UKZN). Phenotypical ESBL-positive isolates were examined for the presence of $b l a_{\mathrm{TEM}}, b l a_{\mathrm{SHV}}, b l a_{\mathrm{CTX}-\mathrm{M}}$, $b l a_{\mathrm{CMY}}, b l a_{\mathrm{DHA}}, b l a_{\mathrm{FOX}}$ and $b l a_{\mathrm{MOX}}$ genes using consensus PCRs (Additional file 1 Table S1) as described [1822]. Briefly, DNA extractions were performed from overnight bacterial cultures using GeneJet Purification kit (Thermo Scientific, USA). The extracted DNA was stored at $-20^{\circ} \mathrm{C}$ until use. PCR products were visualized by agarose gel electrophoresis using UV light after staining with ethidium bromide. $B l a_{\text {СТX-M }}$ amplicons were sent for DNA sequencing at Inqaba Biotec (South Africa).

\section{Genetic typing of ESBL-producing strains by ERIC-PCR}

The clonal relatedness of ESBL-positive isolates was examined by ERIC- PCR and analysed as described [22, 23]. Briefly, PCR products were visualized by agarose gel electrophoresis and genetic variation was analysed using the GelComparII version 6.0 software package (Applied Maths, Belgium) and Unweighted Pair Group Method with Arithmetic Mean (UPGMA) cluster analysis to produce a dendrogram.

\section{Results}

\section{Bacterial isolate selection, identification and AST}

E. coli isolates were recovered from a total number of urinary $(n=199 / 823 ; 24.1 \%)$ and blood $(n=31 / 255$; $12.1 \%)$ culture samples. Urinary tract isolates were from in- $(n=109)$ and out-patients $(n=90)$. All blood culture isolates were from inpatients. A total of 58/199 (29\%) of urine isolates $(51 / 109(47 \%)$ and 7/90 (8\%) isolates from in- and outpatients, respectively showed reduced susceptibility ( $\mathrm{R}$ or I) to cefotaxime and/or ceftazidime and as compared to 17/31 (55\%) of blood isolates (Additional file 1: Tables S2 and S3). The ESBL confirmation test was positive for all $75 / 230$ (32.6\%) isolates tested. The ESBL-positive rates were significantly different in urine isolates from out- versus inpatients, respectively 7/90 (7.7\%) and 51/109 (46.8\%). Reduced susceptibility to meropenem was not observed. MDR was observed in all ESBL-positive isolates.

Reduced susceptibility to cefoxitin was detected in $41 /$ $75(54.6 \%)$ of the isolates tested and further examined for increased AmpC-production; 7/17 and 34/58 were blood and urine isolates, respectively (Tables S2 and S3). A total of $25 / 41$ (60.9\%) isolates were confirmed positive for increased AmpC-production using the ROSCO combined disc method, representing 25/75 (33\%) of the total ESBL-positive population.

The presence of co-resistance to clinically important antibiotics was also examined (Tables S2 and S3) in isolates from blood: ciprofloxacin $(n=12 / 17$ : 71\%), gentamicin $(n=8 / 17$ : 47\% $)$ and trimethoprimsulfamethoxazole $(n=17 / 17 ; 100 \%)$, and urine: ciprofloxacin $(n=40 / 58 ; 69 \%)$, gentamicin $(n=27 / 58 ; 47 \%)$ and trimethoprim-sulfamethoxazole $(n=55 / 58$; $95 \%)$. Interestingly, a majority of the isolates, even pAmpCnegative, expressed resistance to $\beta$-lactamase inhibitors (amoxicillin-clavulanic acid and piperacillin-tazobactam) indicating additional resistance mechanisms.

\section{Detection of genes encoding ESBL and plasmid-mediated AmpC}

A total of 58/75 (77\%), 39/75 (52\%), and $1 / 75(1 \%)$ ESBL-producing isolates were positive for $b l a_{\mathrm{CTX}-\mathrm{M}}$ (Additional file 1: Fig. S1), bla $a_{\mathrm{SHV}}$ and bla $a_{\mathrm{TEM}}$ by PCR, respectively. The $b l a_{\text {СTX-M }}$ amplicons were sequencetyped with the following results: CTX-M-15 $(n=24$; CTX-M group 1), CTX-M-28 ( $n=2$; CTX-M group 1), CTX-M-117 ( $n=1$; CTX-M group 1), CTX-M-36 ( $n=1$; CTX-M-group 1), CTX-M-164 ( $n=1$; CTX-M group 1$)$ and CTX-M no type $(n=29)$ (Fig. S1). Available resources did not allow sequence typing of SHVamplicons, but $13 / 17$ (76.5\%) of bla ${ }_{\mathrm{CTX}-\mathrm{M}^{-} \text {-negative, }}$ ESBL-producing isolates were $b l a_{\mathrm{SHV}}$-positive, indicating a SHV-ESBL-type. The only $b l a_{\text {TEM }}$ positive isolate was positive for bla $a_{\mathrm{CTX}-\mathrm{M}-15}$. A total of $25 / 75$ (33\%) isolates examined scored positive for one or more plasmid mediated AmpC-genes; $b l a_{\mathrm{CMY}}(n=1), b l a_{\mathrm{DHA}}(n=13)$, bla $_{\mathrm{MOX}}(n=22)$, and $\operatorname{bla}_{\mathrm{FOX}}(n=24)$. The isolates contained two $(n=15)$ or three $(n=10)$ pAmpC genes: $b l a_{\mathrm{CMY} ; \mathrm{DHA}}(n=1), b l a_{\mathrm{DHA} ; \mathrm{FOX}}(n=2), b l a_{\mathrm{MOX} ; \mathrm{FOX}}(n=$ $12)$ and $b l a_{\text {DHA;MOX;FOX }}(n=10)$. Importantly, only phenotypical AmpC-isolates were positive for bla AmpC.

\section{ERIC - PCR results}

Distinct fragment length polymorphisms were obtained for the 75 isolates tested and used as a means of differentiating E. coli isolates (Fig. S1). The absence or presence of a band was also examined in deciding divergence in ERIC-PCR profiles. The profiles comprised between 4 and 12 individual bands, varying in size from $0.5-10 \mathrm{~kb}$ (Fig. S1). Visual analysis of the profiles included primary, secondary and tertiary amplification representing different amplification intensities. Primary amplification products refer to fragments of highest intensity on the gels. Secondary amplification products were fragments that are not as intense as the primary amplification products but stronger than the tertiary amplification products, which were the minor amplification products of low intensity. All isolates were typeable 
and band patterns were reproducible on repeat amplification.

The ERIC-PCR profiles allowed separation of the 75 isolates into 50 types grouped into eight main clusters (A-H), and further sub-divided into two or three main sub-clusters (Fig. S1). The urine isolates were diverse and distributed in all eight identified clusters (clusters A-H) with characteristics as outlined below. Overall, isolates with similar profiles demonstrated different $\beta$ lactamase gene content with regard to $b l a_{\mathrm{CTX}-\mathrm{M}^{-}}$allelic profile (Fig. S1). However, strains in cluster B $(n=12)$ including nine strains from patients at the pediatric department showed a CTX-M group 1/-15 profile. Bla $_{\mathrm{SHV}} \quad(n=39)$, and/or blapAmpC-positive $(n=25)$ strains were distributed across clusters without any apparent patterns (data not shown). However, blapAmpCs were detected across seven out of eight clusters (A-G) and only present in strains from inpatients, indicating in-hospital plasmid spread between strains. The blood isolates were more clonal in nature and mostly observed in clusters $F$ and $G$, but predominantly in cluster G. Although closely related, they demonstrated differences in their $\beta$-lactamase resistance gene content (data not shown).

\section{Discussion}

Literature reviews show that most studies on the presence of clinical isolates of ESBL-producing Enterobacterales in Africa have been conducted in Northern and Eastern Africa, with a relative lack of data in SubSaharan Africa [13, 24-26]. In general, and particularly in Mozambique there is very limited data from surveillance or clinical studies documenting the susceptibility pattern of common pathogens. Previous studies have revealed a great variety in proportions of ESBL-producing Enterobacterales, underlining the importance of surveillance studies and local data in order to guide antimicrobial therapy and infection control $[24,25]$.

In this study, the overall prevalence of plasmidmediated AmpC- and/or ESBL-production in clinical isolates of $E$. coli was $10.8 \%(25 / 230)$ and $32.6 \%$ (75/ $230)$, respectively. Both phenotypes were present in urinary tract and blood culture isolates and with a significant higher prevalence of ESBL in urinary isolates obtained from inpatients (8\%) compared to outpatients (47\%). CTX-Ms were the most dominant ESBL-type, with CTX-M group 1 and $b l a_{\mathrm{CTX}-\mathrm{M}-15}$ as the major subtype and allele, respectively. Most of the bla $a_{\text {СтX-M negative, }}$ ESBL-positive isolates were negative for $b l a_{\text {TEM }}$, but positive for $b l a_{\mathrm{SHV}}$, indicating an SHV-ESBL-type. This is in accordance with the international situation [5, 7] and the recent meta-analysis of ESBL-producing Enterobacterales in East Africa hospitals [24].
Moreover, a study of ESBL-producing Enterobacterales in stool samples in Mali, Niger and Cameroon showed that CTX-M was the dominant ESBL-type [26]. Finally, our results are also in line with the recent detection of a relatively high prevalence of ESBL CTX-M-type producing Enterobacterales in stool samples from Mozambique university students [22].

A total of $11 \%$ (25/230) of the isolates expressed an AmpC phenotype and all those were pAmpC-PCR positive. Surprisingly, all isolates contained two to three pAmpC genes of which $b l a_{\mathrm{FOX}}$ was the most prevalent. These observations are in contrast with the worldwide observations of $b l a_{\mathrm{CMY}}$ as the most prevalent pAmpC gene in E. coli populations [8, 9]. CMY-2 has the broadest geographic spread among pAmpCs and is an important cause of extended-spectrum beta-lactam resistance in E. coli as well as in non-typhoid Salmonella strains in many countries [27]. The finding of multiple pAmpCbla genes in single strains has recently been reported in a Tunisian study. Briefly, a total of 11 out of 75 pAmpC positive clinical strains of Enterobacterales were shown to contain up to three different pAmpCs [10]. In contrast to our study, CMY-2 was the most common pAmpC-type in their study. Moreover, the combination of MOX-, FOX- and CMY-2 type enzymes was dominant in their isolates, in contrast to ours that mostly contained MOX- and FOX-types in combination with DHA.

ERIC-PCR has been a useful rapid method in various molecular epidemiological studies to describe the genetic relatedness in Enterobacterales strain collections [28]. Our ERIC-PCR results revealed an overall genetic diversity of pAmpC - and/or ESBL - positive E. coli strains at the Maputo Central Hospital. The results indicate that there is not a dominant clone of ESBL-/pAmpC positive E. coli. However, there are several clusters with clonal relatedness indicating minor outbreaks between patients at specific departments. This notion is supported by the isolation of CTX-M-15 producing strains with similar resistance patterns from the Pediatric department linked in time within cluster B.

The observation of multidrug-resistant pAmpC- and/or ESBL-producing E. coli in a high proportion of clinical isolates during a period of 3 months is a major concern. $E$. coli is the most prevalent cause of urinary tract infections and Gram-negative bacteremia in most countries [29, 30]. A large proportion of the ESBL-producing strains also expressed resistance to fluoroquinolones, aminoglycosides and trimethoprim-sulfamethoxazole, limiting treatment options to last resort antibiotics such as carbapenems, piperacillin-tazobactam, colistin or tigecycline. Those drugs are not easily available at Maputo Central Hospital and in developing countries in general. Recent 2015-data from the Pharmacy Department at Maputo Central Hospital showed that betalactams represented $75 \%$ of the total 
in-house antibiotic use, in which ceftriaxone (a third generation cephalosporin) covered $21 \%$ of the betalactams (Zimba TF et al. unpublished). Carbapenems were not in use in 2015. Thus, a significant proportion of clinical $E$. coli strains at the Maputo Central Hospital is in fact not treatable with the locally available antibiotics. This is in line with the recently reported antimicrobial surveillance data of blood culture Gram-negative pathogens from Blantyre, Malawi [31].

In conclusion, our study has shown: (i) a high proportion of pAmpC-and/or ESBL-producing clinical isolates of E. coli from the urinary tract and blood cultures at the Central Hospital [26]. CTX-Ms and FOX/MOX were the dominant ESBL- and pAmpC-types, respectively. (iii) All ESBL- and pAmpC-producing isolates were multidrug-resistant, only susceptible to antibiotics that are not easily available at the hospital. (iv) Studies of the genetic relatedness between ESBL- and / or pAmpCproducing isolates demonstrate genetic diversity and some clusters indicating within-hospital spread of isolates. The overall findings strongly support the urgent need for accurate and rapid diagnostic services to guide antibiotic treatment of life-threatening infections and improved infection control measures. The findings have a probable transfer value to other hospitals in Mozambique as the Central Hospital has reference functions with transfer of patients to and from other hospitals.

\section{Supplementary Information}

The online version contains supplementary material available at https://doi. org/10.1186/s12879-020-05696-y.

Additional file 1: Table S1. Primer sequences for PCR amplification of ESBL and pAmpC genes. Table S2. Antimicrobial susceptibility for ESBLpositive E. coli blood isolates ( $n=17$ ). Table S3. Antimicrobial susceptibility for ESBL-positive $E$. coli urine isolates $(n=58)$. Figure $\mathbf{S 1}$. Cluster analysis of ESBL-positive E. coli $(n=75)$ isolates based on ERIC-PCR

fingerprinting patterns using Jacquard index and UPGMA clustering. The scale at the top left represents percentage similarity. Isolate columns: identification with origins in brackets: $\mathrm{O}=$ outpatient, $\mathrm{P}=$ paediatrics, $\mathrm{M}=$ medicine, $\mathrm{S}=$ surgery, $\mathrm{U}=$ urine and $\mathrm{B}=$ blood. Presence $(+)$ or absence $(-)$ of bla $a_{C T X-M}$ and sequence type are also indicated for each strain.

\section{Abbrevations}

AST: Antimicrobial susceptibility testing; ATCC: American Type Culture Collection; ERIC-PCR: Enterobacterial repetitive intergenic consensus PCR; ESBL: Extended-spectrum $\beta$-lactamase; ISCISA: Instituto Superior de Ciêncas de Saúde; MCH: Maputo Central Hospital; MDR: Multidrug-resistance; pAmpC: Plasmid-mediated AmpC $\beta$-lactamase; UKZN: University of KwaZuluNatal; UPGMA: Unweighted Pair Group Method with Arithmetic Mean

\section{Acknowledgments}

The authors acknowledge the Norwegian Agency for Development Cooperation for funding this study, the Microbiology Laboratory of Maputo Central Hospital, Maputo, Mozambique for providing isolates, and the Microbiology Laboratory of School of Health Science, University of KwaZuluNatal, Durban, South Africa for providing space and reagents in the accomplishment of the molecular diagnostics.

\section{Authors' contributions}

The study was designed by CELE, TFZ, GSS, BH, SYE, and AS. Collection of data and laboratory analysis were performed by CELE, JOS, UG, and HYC. All authors contributed to the interpretation of data and writing of the manuscript. All authors read and approved the final manuscript.

\section{Funding}

The study was funded by the Norwegian Agency for Development Cooperation under the Norwegian Program for Capacity Development in Higher Education and Research for Development (NORHED) Grant QZA 0484 RSA 13/0010 entitled "Antibiotic Stewardship and Conservancy in Africa". The funding body had no role in the design of the study, collection, analysis, or interpretation of data, or in writing the manuscript.

\section{Availability of data and materials}

The datasets used and/or analysed during the current study are available from the first and the corresponding author on reasonable request.

\section{Ethics approval and consent to participate}

This study was approved by the Biomedical Research Ethics Committee of the University of KwaZulu-Natal (UKZN: BE 030/16) and the Institutional Bioethics Committee for Health-CIBS- Instituto Superior de Ciêncas de Saúde (ISCISA: TFCMCSCE 02/15). The committees waived the requirements for informed consent.

\section{Consent for publication}

Not applicable.

\section{Competing interests}

SYE is a member of the Global Respiratory Infection Partnership sponsored by an unrestricted educational grant from Reckitt and Benckiser, UK.

\section{Author details}

${ }^{1}$ Microbiology Laboratory, Maputo Central Hospital, Maputo, Mozambique. ${ }^{2}$ High Institute of Health Sciences (ISCISA), Maputo, Mozambique. ${ }^{3}$ Antimicrobial Research Unit, School of Health Sciences, University of KwaZulu-Natal, Durban, South Africa. ${ }^{4}$ Discipline of Microbiology, School of Life Sciences, University of KwaZulu-Natal, Durban, South Africa. ${ }^{5}$ Department of Microbiology and Infection Control, Norwegian National Advisory Unit on Detection of Antimicrobial Resistance, University Hospital of North Norway, Tromsø, Norway. ${ }^{6}$ Research Group for Host-Microbe Interaction, Department of Medical Biology, Faculty of Health Sciences, UiT Arctic University of Norway, NO-9037 Tromsø, Norway.

Received: 9 July 2019 Accepted: 8 December 2020

Published online: 06 January 2021

\section{References}

1. Klein EY, et al. Tracking global trends in the effectiveness of antibiotic therapy using the drug resistance index. BMJ Glob Health. 2019;4(2): e001315.

2. Gelband H, Laxminarayan R. Tackling antimicrobial resistance at global and local scales. Trends Microbiol. 2015;23(9):524-6.

3. Russo A, et al. Predictors of outcome in patients with severe sepsis or septic shock due to extended-spectrum beta-lactamase-producing Enterobacteriaceae. Int J Antimicrob Agents. 2018:52(5):577-85.

4. Bush K, Fisher JF. Epidemiological expansion, structural studies, and clinical challenges of new beta-lactamases from gram-negative bacteria. Annu Rev Microbiol. 2011;65:455-78.

5. Woerther PL, et al. Trends in human fecal carriage of extended-spectrum beta-lactamases in the community: toward the globalization of CTX-M. Clin Microbiol Rev. 2013;26(4):744-58.

6. Bush K, Jacoby GA. Updated functional classification of beta-lactamases. Antimicrob Agents Chemother. 2010;54(3):969-76.

7. Naseer U, Sundsfjord A. The CTX-M conundrum: dissemination of plasmids and Escherichia coli clones. Microb Drug Resist. 2011;17(1):83-97.

8. Jacoby GA. AmpC beta-lactamases. Clin Microbiol Rev. 2009;22(1):161-82 Table of Contents.

9. Dolejska M, Papagiannitsis CC. Plasmid-mediated resistance is going wild. Plasmid. 2018;99:99-111. 
10. Cherif T, et al. Cooccurrence of multiple AmpC beta-lactamases in Escherichia coli, Klebsiella pneumoniae, and Proteus mirabilis in Tunisia. Antimicrob Agents Chemother. 2016;60(1):44-51.

11. Mandomando I, et al. Antimicrobial drug resistance trends of bacteremia isolates in a rural hospital in southern Mozambique. Am J Trop Med Hyg. 2010;83(1):152-7.

12. Moon TD, et al. Bacteremia as a cause of fever in ambulatory, HIV-infected Mozambican adults: results and policy implications from a prospective observational study. PLoS One. 2013;8(12):e83591.

13. Leopold SJ, et al. Antimicrobial drug resistance among clinically relevant bacterial isolates in sub-Saharan Africa: a systematic review. J Antimicrob Chemother. 2014;69(9):2337-53.

14. Matuschek E, Brown DF, Kahlmeter G. Development of the EUCAST disk diffusion antimicrobial susceptibility testing method and its implementation in routine microbiology laboratories. Clin Microbiol Infect. 2014;20(4):0255-66.

15. Magiorakos AP, et al. Multidrug-resistant, extensively drug-resistant and pandrug-resistant bacteria: an international expert proposal for interim standard definitions for acquired resistance. Clin Microbiol Infect. 2012;18(3): 268-81.

16. EUCAST, EUCAST guidelines for detection of resistance mechanims and specific resistances of clinical and/or epidemiological importance, in www. eucast.org/resistance mechanisms/. 2017. p. 4-11.

17. Hansen $F$, et al. Evaluation of ROSCO neo-Sensitabs for phenotypic detection and subgrouping of ESBL-, AmpC- and carbapenemaseproducing Enterobacteriaceae. APMIS. 2012;120(9):724-32.

18. Sharma J, Sharma M, Ray P. Detection of TEM \& SHV genes in Escherichia coli \& Klebsiella pneumoniae isolates in a tertiary care hospital from India. Indian J Med Res. 2010;132:332-6.

19. Saladin M, et al. Diversity of CTX-M beta-lactamases and their promoter regions from Enterobacteriaceae isolated in three Parisian hospitals. FEMS Microbiol Lett. 2002;209(2):161-8.

20. Brinas $L$, et al. Detection of CMY-2, CTX-M-14, and SHV-12 beta-lactamases in Escherichia coli fecal-sample isolates from healthy chickens. Antimicrob Agents Chemother. 2003;47(6):2056-8.

21. Perez-Perez FJ, Hanson ND. Detection of plasmid-mediated AmpC betalactamase genes in clinical isolates by using multiplex PCR. J Clin Microbiol. 2002:40(6):2153-62.

22. Chirindze LM, et al. Faecal colonization of E. coli and Klebsiella spp. producing extended-spectrum beta-lactamases and plasmid-mediated AmpC in Mozambican university students. BMC Infect Dis. 2018;18(1):244.

23. Versalovic J, Koeuth T, Lupski JR. Distribution of repetitive DNA sequences in eubacteria and application to fingerprinting of bacterial genomes. Nucleic Acids Res. 1991;19(24):6823-31.

24. Sonda T, et al. Meta-analysis of proportion estimates of extended-SpectrumBeta-lactamase-producing Enterobacteriaceae in East Africa hospitals. Antimicrob Resist Infect Control. 2016:5:18

25. Mshana SE, Matee M, Rweyemamu M. Antimicrobial resistance in human and animal pathogens in Zambia, Democratic Republic of Congo, Mozambique and Tanzania: an urgent need of a sustainable surveillance system. Ann Clin Microbiol Antimicrob. 2013;12:28.

26. Sangare $S A$, et al. Prevalence of extended-spectrum beta-lactamaseproducing Enterobacteriaceae isolated from blood cultures in Africa. Med Mal Infect. 2015;45(9):374-82.

27. Egorova S, et al. Ceftriaxone-resistant salmonella enterica serotype Newport, France. Emerg Infect Dis. 2008;14(6):954-7.

28. Ranjbar $R$, et al. Typing methods used in the molecular epidemiology of microbial pathogens: a how-to guide. New Microbiol. 2014;37(1):1-15.

29. Foxman B. The epidemiology of urinary tract infection. Nat Rev Urol. 2010; 7(12):653-60.

30. de Kraker ME, et al. The changing epidemiology of bacteraemias in Europe: trends from the European antimicrobial resistance surveillance system. Clin Microbiol Infect. 2013;19(9):860-8.

31. Musicha $\mathrm{P}$, et al. Trends in antimicrobial resistance in bloodstream infection isolates at a large urban hospital in Malawi (1998-2016): a surveillance study. Lancet Infect Dis. 2017;17(10):1042-52

\section{Publisher's Note}

Springer Nature remains neutral with regard to jurisdictional claims in published maps and institutional affiliations.

\section{Ready to submit your research? Choose BMC and benefit from:}

- fast, convenient online submission

- thorough peer review by experienced researchers in your field

- rapid publication on acceptance

- support for research data, including large and complex data types

- gold Open Access which fosters wider collaboration and increased citations

- maximum visibility for your research: over $100 \mathrm{M}$ website views per year

At BMC, research is always in progress.

Learn more biomedcentral.com/submissions 